\%

Neurology and Neuroscience

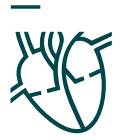

Cardiovascular System

\section{0}

CiteScore: $\mathbf{3 . 1}$

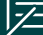

Acceptance rate $48 \%$

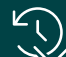

Tme to final decision 9 weeks

\title{
Cerebrovascular Diseases Extra
}

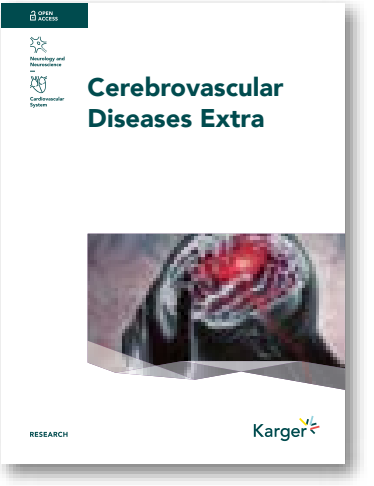

๑ OPEN

Editor-in-Chief

Craig S. Anderson (Sydney, NSW)

\section{Clinical Practice and Policy in Cerebrovascular Diseases}

Cerebrovascular Diseases Extra is an Open Access and online-only journal that focuses on informing the practice of stroke medicine and patient care in different world regions. This journal accepts original research papers, reviews, case series of particular interest, commentaries on clinical topics, case-based ethical issues, practice management tips, novel systems of care, and health policy discussions. Cerebrovascular Diseases Extra publishes content based on both independent submissions and submissions transferred from Cerebrovascular Diseases.

editorialoffice_cee@

karger.com

karger.com/cee

Twitter 